\title{
Effect of Brain-Cor Injection of Ginkgo on the Hyperlipemia and Thrombosis: Fundamental Study
}

\section{Hong YC*, Jo KH and So CH}

Department of Obstetrics and Gynecology, Pyongyang University of Medical Sciences, Democratic People's Republic of Korea

*Corresponding author: Hong YC, Department of Obstetrics and Gynecology, Pyongyang University of Medical Sciences, Democratic People's Republic of Korea

Received: August 20, 2021; Accepted: September 17, 2021; Published: September 24, 2021

\begin{abstract}
We have done this study to verify effects on the serum lipid content (TC, TG, HDL-C) in experimental model of hyperlipemia and on the blood coagulability in experimental model of thrombosis.
\end{abstract}

Background: Female hormone preparation is limited in long term use because of hyperlipemia and thrombosis that are complications occurring in long term use of female hormone preparations for dysfunctional uterine bleeding, climacteric disorder, female genital organs tumor. Therefore to prevent complications such as hyperlipemia and thrombosis with female hormone preparation use are the acute problems not only for the effective treatment of gynecological diseases but also for the lowering of incidence rate of hypertension, arteriosclerosis, hyperlipemia and thrombosis.

We made brain-cor injection of Ginkgo for the treatment of hyperlipemia and thrombosis that are complications occurring in long term use of female hormone preparations and verified its effects on the serum lipid content in experimental model of hyperlipemia and on the blood coagulability in experimental model of thrombosis.

Objects and Materials: Wistar rats with 160 180 g in body weight were used as experimental animals, brain-cor injection of Ginkgo $(70 \mathrm{mg} / 20 \mathrm{~mL})$ made from Ginkgo extract that consists of flavonoid content above $24 \%$, total lactone content above $6 \%$ and total ginkgolic acid content below $5 \mu \mathrm{g} / \mathrm{g}$ was used as drugs.

Methods: For 15 days brain-cor injection of Ginkgo $10 \mathrm{mg} / \mathrm{kg}$ was injected in abdominal cavity of experimental model of hyperlipemia by tween-80, serum was separated from collected blood in orvita vein, serum TC (Total Cholesterol) content, serum TG (Triglyceride) content and serum HDL-C (High Density Lipoprotein-Cholesterol) content were examined with blood automatic biochemical analyzer and measurements were compared each other. For 15 days brain-cor injection of Ginkgo $10 \mathrm{mg} / \mathrm{kg}$ was injected in abdominal cavity of experimental model of hyperlipemia and thromboplastin $0.5 \% 0.1 \mathrm{~mL} / 10 \mathrm{~g}$ was injected on cauda vein in an hour after the last injection of Ginkgo. BT (Bleeding Time), CT (Clotting Time), APTT (Activated Partial Thromboplastin Time) and PT (Prothrombin Time) were examined and measurements were compared each other.

Results: Brain-cor injection of Ginkgo decreased serum TC and TG content and increased serum HDL-C content in experimental model of hyperlipemia. And brain-cor injection of Ginkgo prolonged BT, CT, APTT and PT significantly in experimental model of thrombosis.

Keywords: Female hormone preparation; Hyperlipemia; Thrombosis; Brain-cor injection of Ginkgo

\section{Abbreviations}

TC: Total Cholesterol; TG: Triglyceride; HDL-C: High Density Lipoprotein-Cholesterol; BT: Bleeding Time; CT: Clotting Time; APTT: Activated Partial Thromboplastin Time; PT: Prothrombin Time

\section{Introduction}

Up to now a lot of studies for the treatment of disease with ginkgo leaves have been progressed and in the process of study medicines and healthy food such as Ginkgo pills for arteriosclerosis, cor injection of Ginkgo, cor tincture of Ginkgo and Ginkgo tea were standardized and used. But these medicines and healthy food were prepared from Ginkgo extract with only flavonoid content above $24 \%$, but not lactone components, therefore they do not display enough therapeutic effect.

Worldwide standardized and used Ginkgo extract consists of flavonoid content above $24 \%$, total lactone content above $6 \%$ and total ginkgolic acid content below $5 \mu \mathrm{g} / \mathrm{g}$. This Ginkgo extract is acknowledged as natural medicine that is effective in the treatment
Austin J Obstet Gynecol - Volume 8 Issue 7 - 2021

Submit your Manuscript | www.austinpublishinggroup.com

Hong et al. (c) All rights are reserved
Citation: Hong $\mathrm{YC}$, Jo $\mathrm{KH}$ and So $\mathrm{CH}$. Effect of Brain-Cor Injection of Ginkgo on the Hyperlipemia and Thrombosis: Fundamental Study. Austin J Obstet Gynecol. 2021; 8(7): 1193. 
of cardiovascular and nervous system diseases such as cerebral apoplexy, angina pectoris, hypertension, hyperlipemia, senile dementia, diabetes mellitus and has few side effects. Especially it is known that lactone component in Ginkgo extract is a strong natural inhibitor to platelet-activating factors. At present Ginkgo preparation is developing into one of the medicines in high demand in the treatment of cardiovascular and nervous system diseases.

On the other hand female hormone preparation is limited in long term use because of hyperlipemia and thrombosis that are complications occurring in long term use of female hormone preparations for dysfunctional uterine bleeding, climacteric disorder, female genital organs tumor. Therefore to prevent complications such as hyperlipemia and thrombosis with female hormone preparation use is becoming an acute problem not only for the effective treatment of gynecological diseases but also for the lowering of incidence rate of hypertension, arteriosclerosis, hyperlipemia and thrombosis.

\section{Objects and Methods}

\section{Objects}

Wistar rats with 160 180 g in body weight were used as experimental animals.

\section{Materials}

Brain-cor injection of Ginkgo $(70 \mathrm{mg} / 20 \mathrm{~mL})$ made from Ginkgo extract that consist of flavonoid content above $24 \%$, total lactone content above $6 \%$ and total ginkgolic acid content below $5 \mu \mathrm{g} / \mathrm{g}$ was used as drugs for study group, and cor injection of Ginkgo made from Ginkgo extract with only flavonoid was used as drugs for control group.

\section{Methods}

Methods to examine the effect of brain-cor injection of Ginkgo on serum TC, TG and HDL-C content: Experimental model of hyperlipemia (control group) was made by injection of tween-80 1g/ $\mathrm{kg}$ in abdominal cavity of rat five times every other day. Study group No 1 was administrated cor injection of Ginkgo (control drug) and study group No 2 was administrated brain-cor injection of Ginkgo (study drug). Study groups were administrated injections $10 \mathrm{mg} / \mathrm{kg}$ in abdominal cavity for 15 days. Normal group were not administrated any drugs. Serum was separated from collected blood in orvita vein, serum TC(total cholesterol) content, serum TG (Triglyceride) content and serum HDL-C (high density lipoprotein-cholesterol) content were examined with blood automatic biochemical analyzer, and measurements were compared each other.

Methods to examine the effect of brain-cor injection of Ginkgo on blood coagulability: Cor injection of Ginkgo and brain-cor injection of Ginkgo were injected $10 \mathrm{mg} / \mathrm{kg}$ in dose for 15 days in abdominal cavity of experimental model belonging to the study group
No 1 and No 2 respectively, thromboplastin $0.5 \% \quad 0.1 \mathrm{~mL} / 10 \mathrm{~g}$ was injected on cauda vein in an hour after the last injection of Ginkgo. Control group was injected only thromboplastin and normal group was not administrated any drugs. Some parameters for coagulation were examined and compared each other. BT (Bleeding Time) was examined by Duke method, CT (Clotting Time) was examined by Zali-Ponio method, APTT (Activated Partial Thromboplastin Time) was examined by kaolin-cephalin method, PT (Prothrombin Time) was examined by micro rapid method.

\section{Results}

Effect of brain-cor injection of Ginkgo on serum lipid content in experimental model of hyperlipemia

Effect of brain-cor injection of Ginkgo on serum TC content in experimental model of hyperlipemia: As shown in Table 1, TC content was $126.8 \pm 6.2 \mathrm{mg} / \mathrm{dl}, 137.1 \pm 6.6 \mathrm{mg} / \mathrm{dl}$ in study group No 2 and No 1 respectively, and TC content of study groups decreased in contrast with control group $(168.5 \pm 8.8 \mathrm{mg} / \mathrm{dl})$ significantly (respectively $\mathrm{P}<0.01, \mathrm{P}<0.05$ ). There was no difference of TC content between study group No 1 and No 2, but TC content of study group No 2 decreased more in contrast with study group No 1 .

Effect of brain-cor injection of Ginkgo on serum TG content in experimental model of hyperlipemia: As shown in Table 2, TG content was $163.5 \pm 11.9 \mathrm{mg} / \mathrm{dl}, 178.4 \pm 12.7 \mathrm{mg} / \mathrm{dl}$ in study group No 2 and No 1 respectively, and TG content of study groups decreased in contrast with control group $(235.6 \pm 15.6 \mathrm{mg} / \mathrm{dl})$ significantly (respectively $\mathrm{P}<0.01, \mathrm{P}<0.05$ ). There was no difference of $\mathrm{TG}$ content between study group No 1 and No 2, but TG content of study group No 2 decreased more in contrast with study group No 1.

Effect of brain-cor injection of Ginkgo on serum HDL-C content in experimental model of hyperlipemia: As shown in Table 3 , HDL-C content was $36.5 \pm 1.6 \mathrm{mg} / \mathrm{dl}, 33.1 \pm 1.3 \mathrm{mg} / \mathrm{dl}$ in study group No 2 and No 1 respectively, and HDL-C content of study groups increased in contrast with control group $(27.2 \pm 1.4 \mathrm{mg} / \mathrm{dl})$ significantly $(\mathrm{P}<0.01)$. There was no difference of HDL-C content between study group No 1 and No 2, but HDL-C content of study group No 2 increased more in contrast with study group No 1.

\section{Effect of brain-cor injection of Ginkgo on blood coagulability in experimental model of thrombosis}

Effect of brain-cor injection of Ginkgo on BT and CT in experimental model of thrombosis: As shown in Table 4, BT was $77.0 \pm 5.9$ seconds, $72.0 \pm 5.1$ seconds in study group No 2 and No 1 respectively, and $\mathrm{BT}$ of study groups was prolonged in contrast with control group $(58.0 \pm 4.2$ seconds) significantly $(\mathrm{P}<0.05)$. There was no difference of BT between study group No 1 and No 2, but BT of study group No 2 was prolonged more in contrast with study group No 1.

Table 1: Effect of brain-cor injection of Ginkgo on serum TC content in experimental model of hyperlipemia $(\bar{x} \pm S E)$.

\begin{tabular}{|c|c|c|c|}
\hline Groups & Drugs & Dose (mg/kg) & Number of rats (heads) \\
\hline Normal group & - & - & 10 \\
\hline Control group & - & - & $111.4 \pm 5.3$ \\
\hline Study group No 1 & Cor injection of Ginkgo & 10 & $108.5 \pm 8.8$ \\
\hline Study group No 2 & Brain-cor injection of Ginkgo & 10 & 10 \\
\hline
\end{tabular}

${ }^{*} \mathrm{p}<0.05 ;{ }^{* *} \mathrm{p}<0.01$ (comparison with control group). 
Table 2: Effect of brain-cor injection of Ginkgo on serum TG content in experimental model of hyperlipemia $(\bar{X} \pm S E)$.

\begin{tabular}{|c|c|c|c|}
\hline Groups & Drugs & Dose (mg/kg) & Number of rats (heads) \\
\hline Normal group & - & - & 10 \\
\hline Control group & - & - & $135.8 \pm 7.6$ \\
\hline Study group No 1 & Cor injection of Ginkgo & 10 & $235.6 \pm 15.6$ \\
\hline Study group No 2 & Brain-cor injection of Ginkgo & 10 & 10 \\
\hline
\end{tabular}

"p<0.05; " $\mathrm{p}<0.01$ (comparison with control group).

Table 3: Effect of brain-cor injection of Ginkgo on serum HDL-C content in experimental model of hyperlipemia ( $\bar{X} \pm$ SE).

\begin{tabular}{|c|c|c|c|c|}
\hline Groups & Drugs & Dose (mg/kg) & Number of rats (heads) & HDL-C (mg/dl) \\
\hline Normal group & - & - & 10 & $41.6 \pm 2.1$ \\
\hline Control group & - & - & 10 & $27.2 \pm 1.4$ \\
\hline Study group No 1 & Cor injection of Ginkgo & 10 & 10 & $33.1 \pm 1.3^{* *}$ \\
\hline Study group No 2 & Brain-cor injection of Ginkgo & 10 & 10 & $36.5 \pm 1.6^{* *}$ \\
\hline
\end{tabular}

*p<0.01(comparison with control group).

Table 4: Effect of brain-cor injection of Ginkgo on BT and CT in experimental model of thrombosis ( $\bar{x} \pm \mathrm{SE})$.

\begin{tabular}{|c|c|c|c|c|c|}
\hline Groups & Drugs & Dose (mg/kg) & Number of rats (heads) & BT (S) & CT (S) \\
\hline Normal group & - & - & 10 & $94.7 \pm 7.6$ & $237.0 \pm 21.8$ \\
\hline Control group & - & - & 10 & $58.0 \pm 4.2$ & $104.0 \pm 11.2$ \\
\hline Study group No 1 & Cor injection of Ginkgo & 10 & 10 & $72.0 \pm 5.1^{*}$ & $168.0 \pm 12.3^{* *}$ \\
\hline Study group No 2 & Brain-cor injection of Ginkgo & 10 & 10 & $77.0 \pm 5.9^{*}$ & $186.0 \pm 16.7^{\text {t* }}$ \\
\hline
\end{tabular}

${ }^{*} \mathrm{p}<0.05 ;{ }^{* *} \mathrm{p}<0.01$ (comparison with control group).

Table 5: Effect of brain-cor injection of Ginkgo on APTT and PT in experimental model of thrombosis ( $\bar{x} \pm$ SE).

\begin{tabular}{|c|c|c|c|c|}
\hline Groups & Drugs & Dose (mg/kg) & Number of rats (heads) & APTT (S) \\
\hline Normal group & - & - & 10 & $33.3 \pm 1.9$ \\
\hline Control group & - & - & 10 & $18.7 \pm 1.5$ \\
\hline Study group No 1 & Cor injection of Ginkgo & 10 & $13.3 \pm 1.4$ \\
\hline Study group No 2 & Brain-cor injection of Ginkgo & 10 & 10 & $25.2 \pm 1.7^{*}$ \\
\hline
\end{tabular}

" $\mathrm{p}<0.05$; " $\mathrm{p}<0.01$ (comparison with control group).

And CT was $186.0 \pm 16.7$ seconds, $168.0 \pm 12.3$ seconds in study group No 2 and No 1 respectively, and CT of study groups was prolonged in contrast with control group (104.0 \pm 11.2 seconds) significantly $(\mathrm{P}<0.01)$. There was no difference of CT between study group No 1 and No 2, but CT of study group No 2 was prolonged more in contrast with study group No 1.

Effect of brain-cor injection of Ginkgo on APTT and PT in experimental model of thrombosis: As shown in Table 5, APTT was $28.5 \pm 1.6$ seconds, $25.2 \pm 1.7$ seconds in study group No 2 and No 1 respectively, and $\mathrm{BT}$ of study groups was prolonged in contrast with control group (18.71.5 seconds) significantly (respectively $\mathrm{P}<0.01$, $\mathrm{P}<0.05)$. There was no difference of APTT between study group No 1 and No 2, but APTT of study group No 2 was prolonged more in contrast with study group No 1 .

And PT was $21.2 \pm 2.1$ seconds, $18.8 \pm 1.8$ seconds in study group No 2 and No 1 respectively, and PT of study groups were prolonged in contrast with control group ( $13.3 \pm 1.4$ seconds) significantly (respectively $\mathrm{P}<0.01, \mathrm{P}<0.05$ ). There was no difference of $\mathrm{PT}$ between study group No 1 and No 2, but PT of study group No 2 was prolonged more in contrast with study group No 1.

\section{Discussion}

Ginkgo leaves are rich in natural resources and its therapeutic effects are well-advertised. Up to now numerous studies for the treatment of disease with ginkgo leaves were progressed and in the process of study medicines and health food such as Ginkgo pills for arteriosclerosis, cor injection of Ginkgo, cor tincture of Ginkgo and Ginkgo tea are standardized and used. But these medicines and health food were prepared from Ginkgo extract with only flavonoid content above $24 \%$ without lactone components. Therefore these do not display enough therapeutic value.

Worldwide standardized and used Ginkgo extract consists of flavonoid content above $24 \%$, total lactone content above $6 \%$ and total ginkgolic acid content below $5 \mu \mathrm{g} / \mathrm{g}[1,2]$. This Ginkgo extract is acknowledged as natural medicines that are effective in the treatment of cardiovascular and nervous system diseases such as cerebral apoplexy, angina pectoris, hypertension, hyperlipemia, senile dementia, diabetes mellitus and has few side effects $[3,4]$. Especially it is known that lactone component in Ginkgo extract is a strong natural inhibitor to platelet-activating factors. At present Ginkgo preparation has become one of the medicines in high demand in the treatment of cardiovascular and nervous system diseases $[5,6]$.

On the other hand female hormone preparation is limited in long term use because of hyperlipemia and thrombosis that are complications occurring in long term use of female hormone preparations for dysfunctional uterine bleeding, climacteric 
disorder, female genital organs tumor [7-12]. Therefore to prevent complications such as hyperlipemia and thrombosis with female hormone preparation use are the acute problems not only for the effective treatment of gynecological diseases but also for the lowering of incidence rate of hypertension, arteriosclerosis, hyperlipemia and thrombosis.

We made brain-cor injection of Ginkgo for the treatment hyperlipemia and thrombosis that are complications occurring in long term use of female hormone preparations. Our brain-cor injection of Ginkgo consists of flavonoid content above $24 \%$ and total lactone content above $6 \%$ and total ginkgolic acid content below 5 $\mu \mathrm{g} / \mathrm{g}$. We verified its effects on serum lipid content in experimental model of hyperlipemia and on blood coagulability in experimental model of thrombosis.

According to our research data, brain-cor injection of Ginkgo decreased serum TC and TG content and increased serum HDL-C content in experimental model of hyperlipemia. On the other hand brain-cor injection of Ginkgo showed more therapeutic effect on hyperlipemia in contrast with cor injection of Ginkgo used previously. And serum TC and TG content, serum HDL-C content with braincor injection of Ginkgo did not reach normal value, but it tended to reach the value similar to normal value.

According to our research data, brain-cor injection of Ginkgo prolonged BT, CT, APTT and PT significantly in experimental model of thrombosis. On the other hand brain-cor injection of Ginkgo showed more therapeutic effect on thrombosis in contrast with cor injection of Ginkgo used previously. And some parameters for coagulation with brain-cor injection of Ginkgo did not reach normal value, but it tended to reach the value similar to normal value. We recognized that the results mentioned above depend on braincor injection of Ginkgo, because it contains not only flavonoid but also lactone above 6\%. And we predict that using Ginkgo extract containing lactone components as medicinal substances is a way for the improvement of therapeutic effects because lactone components have strong antithrombotic and antihyperlipemic action.

Therefore our brain-cor injection of Ginkgo can be used for the treatment of hyperlipemia and thrombosis that are complications occurring in long term use of female hormone preparations for dysfunctional uterine bleeding, climacteric disorder, female genital organs tumor. However the study for clinical application of this medicine must be continued in future.

\section{Conclusion}

Brain-cor injection of Ginkgo decreased serum TC and TG content and increased serum HDL-C content in experimental model of hyperlipemia, and brain-cor injection of Ginkgo decreased blood coagulability in experimental model of thrombosis significantly.

\section{References}

1. Mark B. Ginkgolic acid levels in ginko leaf extracts. Herral gram. 1997; 41: 29-30.

2. Schwabe KP. Extract from Ginkgo biloba leaves, its method of preparation and pharmaceuticals containing the extract. 1991.

3. Fang LQ. Research progress of Ginkgo biloba preparation form. China Pharmacy. 2007; 18: 1908-1909.

4. Xie Y, Zhang JS. General situation of Ginkgo biloba extract preparations. Chinese Traditional and Herbal Drugs. 2005; 36: 1267-1269.

5. Braquet PG. Platelet-activating factor antagonists isolated from Ginkgo biloba; chemistry, pharmacology and clinical applications. Drugs of the future. 1987; 12: 634-639.

6. Giuseppe M. Role of platelet-activating factor in cardiovascular pathophysiology. Physiological Reviews. 2000; 80: 1669-1699.

7. American College of Obstetricians and Gynecologists. Use of hormonal contraception in women with coexisting medical conditions. Practice Bulletin. 2006; 73: reaffi rmed 2008b.

8. Gaffield ME, Culwell KR, Lee CR. The use of hormonal contraception among women taking anticonvulsant therapy. Contraception. 2011; 83: 16.

9. Jick SS, Hernandez RK. Risk of non-fatal venous thromboembolism in women using oral contraceptives containing drospirenone compared with women using oral contraceptives containing levonorgestrel: case-control study using United States claims data. BMJ. 2011; 342: d2151.

10. Lopez LM, Grimes DA, Chen-Mok M. Hormonal contraceptives for contraception in overweight or obese women. Cochrane Database Syst Rev. 2010; 7: CD008452.

11. Mac Clellan LR, Giles W, Cole J. Probable migraine with visual aura and risk of ischemic stroke: the stroke prevention in young women study. Stroke. 2007; 38: 2438

12. Parkin L, Sharples K, Hernandez RK. Risk of venous thromboembolism in users of oral contraceptives containing drospirenone or levonorgestrel: nested case-control study based on UK General Practice Research Database. BMJ. 2011 ; 342: d2139. 